\title{
Some results on fixed points and best approximation in partial idempotent-valued metric spaces
}

\author{
M. Iranmanrsh, F. Soleimany, S. Radenović
}

\begin{abstract}
In this work, we introduce the concept of a partial idempotent-valued metric space and establish some existence results of fixed point for different contractive type mappings on such spaces. We also study the existence and uniqueness of best proximity points in the setting these spaces.
\end{abstract}

Keywords: Partial metric space, Fixed points, Best approximation

\section{Introduction}

The Banach Contraction Principle states that, if a self-mapping $T$ of a complete metric space $X$ is a contraction mapping, then $T$ has a unique fixed point. In general, this principle has been generalized in two directions. On the one side, the usual contractive (expansive) condition is replaced by a weakly contractive (expansive) condition. On the other side, the action spaces are replaced by metric spaces endowed with an ordered or partially ordered structure. For example, OŔegan and Petrusel and Caballero started the investigations concerning a fixed point theory in ordered metric spaces([11, 4]). Matthews (1994) introduced a new distance on a non-empty set $X$, which is a called partial metric. A partial metric space is an attempt to generalize the metric space by replacing the condition $d(x, x)=0$ with the condition $d(x, x) \leq d(x, y)$ for all $x, y$ in the definition of the metric (See [8]). Very recently, the authors have focused on this subject and have generalized some fixed point theorems from the class of metric spaces to the class of partial metric spaces (See, $[2,10])$. Later, many authors generalized their fixed point theorems on different type of metric spaces $[1,9,13,14,15,20,21]$. In [6] Huang and Zhang replacing the set of real numbers by an ordered Banach space. In [22] the authors studied the operator-valued metric spaces and gave some fixed point theorems on the spaces.

Manuscript received July 20, 2018; accepted February 15, 2019.

M. Iranmanrsh and F. Soleimany are with the Department of mathematical sciences, Shahrood University of Techology, Shahrood, Iran; S. Radenović is with the Faculty of Mechanical Engineering University of Belgrade, Belgrade, Serbia 
Fixed point theory is indispensable for solving various equations of the form $T x=x$ for self-mappings $T$ defined on subsets of metric spaces. Given non-empty subsets $A$ and $B$ of a metric space and a non-self mapping $T: A \rightarrow B$, the equation $T x=x$ does not necessarily have a solution, which is known as a fixed point of the mapping $T$. However, in such circumstances, it may be speculated to determine an element $x$ for which the error $d(x, T x)$ is minimum, in which case $x$ and $T x$ are in close proximity to each other. The best approximation theorems and best proximity point theorems are relevant in this perspective. Many authors have derived extensions of the best approximation theorem in many directions such as Prolla [12], Reich [16], Sehgal and Singh [17], Vetrivel et al.[19], Eldred and Veeramani [5], Mongkolkeha and Kumam [7] and Sadiq Basha and Veeramani [18]. In this paper, we studied a new type partial valued metric space and gave some fixed point theorems in these spaces. The paper is organized as follows: We first introduce a concept of partial idempotent-valued metric space. Moreover, some fixed point theorems for mappings satisfying the contractive on idempotent-valued metric space is established. We give examples were not contractive type mappings respect to metric space but are idempotent-valued contractive mapping. Finally, we shall give some results about best proximity points of cyclic contractions in these spaces.

\section{Preliminaries}

In this section, we shall define the partial idempotent valued metric space and give some properties.

Definition 2.1. A idempotent space is a vector space $K$ over filed $\mathbb{R}$ in which a two fold $(K, \oplus)$ satisfies the following conditions:

(i) $a \oplus(b \oplus c)=(a \oplus b) \oplus c$ for $a, b, c \in K$.

(ii) $a \oplus a=a$, for all $a \in K$.

A idempotent space is commutative if $a \oplus b=b \oplus a$ for $a, b \in K$.

Definition 2.2. Let $(K, \oplus)$ be a idempotent space, we shall employ the canonical order relation $\leq_{\oplus}$ on $K$ defined by

$$
a \leq_{\oplus} b \Leftrightarrow a \oplus b=b .
$$

We shall also write $b \geq_{\oplus} a$ instead of $a \leq_{\oplus} b$.

Example 2.3. Let $K=\mathbb{R}$ with $a \oplus b:=\max \{a, b\}$ or $a \oplus b:=\min \{a, b\}$ for $a, b \in \mathbb{R}$ is idempotent space. 
Example 2.4. Consider matrices having entries in $(\mathbb{R}, \oplus)$. For conforming matrices $A=$ $\left(a_{i j}\right), B=\left(b_{i j}\right)$ matrix addition together with multiplication by a scalar $\lambda \in \mathbb{R}$ follow the conventional rules

$$
\{A+B\}_{i j}=a_{i j} \oplus b_{i j}, \text { and }\{\lambda A\}=\lambda a_{i j}
$$

is idempotent space.

Definition 2.5. Let $(K, \leq)$ be a partially ordered set and $P \subseteq K$, such that $P \neq \emptyset$. An element $a \in P$ is called a maximal (resp. minimal) of $P$, if there exists no $a \neq x \in P$ such that $a<x$ (resp. $x<a$ ). The set of all maximal (resp. minimal) elements of $P$ is denoted by $\max (P)$ (resp. $\min (P)$ ). If for any finite subset $P, \max (P)$ and $\min (P)$ always exists and unique then $K$ is called a totally lattice.

Example 2.6. $\mathbb{R}$ is totally lattice respect to order $\leq_{\oplus}$ which is defined in Example 2.3 .

Example 2.7. Let $S=\{a\}$, and $X=P(S)=\{\emptyset, S\}$ with the inclusion relation $\subseteq$ is a totally lattice. But if $S=\{a, b\}$ then $X$ is not totally lattice.

Example 2.8. Let $X=\mathbb{R}^{2}$ where $(a, b) \leq(c, d)$ if and only if either $a<c$ or $a=c$ and $b \leq d$ is totally lattice.

Definition 2.9. Let $(K, \leq)$ be a partial ordered vector space. Let $\left\{x_{n}\right\}$ be a sequence in $K$ and $x \in K$. If for every $0_{K}<c$, there is $n_{0}$ such that for all $n>n_{0}, x_{n}-x<c$, then $\left\{x_{n}\right\}$ is said to be convergent and $\left\{x_{n}\right\}$ converges to $x$, and $\mathrm{x}$ is the limit of $\left\{x_{n}\right\}$. We denote this by $\lim _{n \rightarrow \infty} x_{n}=x$ or, $x_{n} \rightarrow x$ as $n \rightarrow \infty$.

Definition 2.10. Let $(K, \leq)$ be a partial order vector space, we say the order relation on $K$ has positive cone ordering property if vector $0_{K} \leq a \leq b$ and scalar inequalities $0 \leq r \leq c$ imply that the inequalities

$$
0_{K} \leq r a \leq r b, \quad r x \leq c x .
$$

for all $0_{K} \leq x \in K$.

Definition 2.11. Let $(K, \leq)$ be a partial order vector space. We say $K$ is a normal space if the order relation on $K$ has positive cone ordering property.

Example 2.12. Let $K=\mathbb{R}$ with $a \oplus b:=\max \{a, b\}$ for $a, b \in \mathbb{R}$. It is trivial that $K$ is a normal space.

Throughout this paper, $(K, \oplus)$ is denotes a commutative idempotent space such that $K$ is a totally lattice respect to order $\leq_{\oplus}$. Now with the help of this $\leq_{\oplus}$ one can give the definition of a partial idempotent-valued metric space.

For later applications, it will be convenient to use the notation

$$
K^{+}:=\left\{a \in K: a \geq_{\oplus} 0_{K}\right\}
$$


Definition 2.13. Let $X$ be a non-empty set. Consider the mapping $d: X \times X \rightarrow K^{+}$satisfies:

(i) $d(x, x) \leq_{\oplus} d(x, y)$ for all $x, y \in X$.

(ii) $x=y \Leftrightarrow d(x, x)=d(x, y)=d(y, y)$.

(iii) $d(x, y)=d(y, x)$ for all $x, y \in X$,

(v) $d(x, y) \leq_{\oplus} d(x, z) \oplus d(y, z)$ for all $x, y, z \in X$.

Then $d$ is called a partial idempotent-valued metric on $X$ and $(X, K, d)$ is called a partial idempotent-valued metric space.

Example 2.14. Let $X=[0, \infty), K=\mathbb{R}$ with the operations $a \oplus_{\max } b:=\max \{a, b\}$. Define the metric $d: X \times X \rightarrow K^{+}$by

$$
d(a, b):=a \oplus b .
$$

Then $X$ is a partial idempotent-valued metric space.

Example 2.15. Let $M$ be a nonempty set and $X=B\left(M, \mathbb{R}^{+}\right)$be the set of bounded mappings(mappings with order-bounded range). Let $K=B\left(X,\left(\mathbb{R}, \oplus_{\max }\right)\right)$, with the pointwise generalized addition $(h \oplus g)(a)=h(a) \oplus g(a)$ on $X$. Define the metric mapping $d:=X \times X \rightarrow K^{+}$by

$$
d(f, g)(a):=\max \{f(a), g(a)\} .
$$

Then $(X, d)$ is a partial idempotent-valued metric space.

Definition 2.16. Let $(X, K, d)$ be a partial idempotent-valued metric space.

(i) a sequence $\left\{x_{n}\right\} \subseteq X$ converges to $x \in X$ if and only if

$$
d(x, x)=\lim _{n \rightarrow \infty} d\left(x, x_{n}\right)=\lim _{n \rightarrow \infty} d\left(x_{n}, x\right)
$$

(ii) a sequence $\left\{x_{n}\right\} \subseteq X$ is called a Cauchy sequence if and only if for $n, m \in N, \lim _{n, m \rightarrow \infty} d\left(x_{n}, x_{m}\right)$ exists.

iii) the $(X, d)$ is said to be complete if every Cauchy sequence $\left\{x_{n}\right\} \subseteq X$ converges, to a point $x \in X$ such that

$$
d(x, x)=\lim _{n, m \rightarrow \infty} d\left(x_{m}, x_{n}\right)=\lim _{n, m \rightarrow \infty} d\left(x_{n}, x_{m}\right) .
$$

Definition 2.17. Let $C$ be a subset of $X$. If for any sequence $\left\{x_{n}\right\} \in X$, there is a subsequence $\left\{x_{n_{i}}\right\}$ of $\left\{x_{n}\right\}$ such that $\left\{x_{n_{i}}\right\}$ is convergent in $C$. Then $C$ is called a sequentially compact set.

Definition 2.18. Suppose that $(X, K, d)$ be a partial idempotent-valued metric space. $T$ : $X \rightarrow X$ is called a continuous function at $x$ if for any $x_{n} \rightarrow x$ implies that $T x_{n} \rightarrow T x$. 


\section{Main results}

In this section, we shall prove some fixed point theorems for contractive mappings in the setting of partial idempotent-valued metric spaces.

Theorem 3.1. Let $X$ be complete partially idempotent-valued metric space and $T: X \rightarrow X$ is a mapping such that

$$
d(T x, T y) \leq_{\oplus} \psi(\max \{d(x, T x), d(x, y), d(y, T y)\}) .
$$

for all $x, y \in X$, where $\psi: K^{+} \rightarrow K^{+}$is a continuous, nondecreasing function such that $\lim _{n \rightarrow \infty} \psi^{n}(a)=0_{K}$ and $\psi(a)<_{\oplus}$ a for $a \in K^{+}$. Then there exists unique $x \in X$ such that $x=T x$.

Proof. Now, let $x_{n}=T x_{n-1}$ for $n=1,2, \ldots$ If $x_{n_{0}}=x_{n_{0}-1}$ for some $n_{0} \in N$, then it is clear that $x_{n_{0}}$ is a fixed point of $T$. Thus, assume $x_{n_{0}} \neq x_{n_{0}-1}$ for all $n$.

$$
\begin{aligned}
d\left(x_{n}, x_{n+1}\right) & \left.=d\left(T x_{n-1}, T x_{n}\right)\right) \\
\leq & \psi\left(\max \left\{d\left(x_{n-1}, T x_{n-1}\right), d\left(x_{n-1}, x_{n}\right), d\left(x_{n}, T x_{n}\right)\right\}\right) . \\
& =\psi\left(\max \left\{d\left(x_{n-1}, x_{n}\right), d\left(x_{n}, x_{n+1}\right)\right\}\right) .(*)
\end{aligned}
$$

Now, if

$$
\max \left\{d\left(x_{n-1}, x_{n}\right), d\left(x_{n}, x_{n+1}\right)\right\}=d\left(x_{n}, x_{n+1}\right)
$$

for some $\mathrm{n}$, then from $(*)$ we have

$$
d\left(x_{n}, x_{n+1}\right) \leq_{\oplus} \psi\left(d\left(x_{n+1}, x_{n}\right)\right)<_{\oplus} d\left(x_{n+1}, x_{n}\right) .
$$

which is a contradiction. Thus

$$
\max \left\{d\left(x_{n-1}, x_{n}\right), d\left(x_{n}, x_{n+1}\right)\right\}=d\left(x_{n}, x_{n-1}\right),
$$

for all $\mathrm{n}$. Therefore, we have

$$
d\left(x_{n}, x_{n+1}\right) \leq_{\oplus} \psi\left(d\left(x_{n-1}, x_{n}\right)\right),
$$

and so

$$
d\left(x_{n}, x_{n+1}\right) \leq_{\oplus} \psi^{n}\left(d\left(x_{0}, x_{1}\right)\right) .
$$

This shows that $\lim _{n \rightarrow \infty} d\left(x_{n}, x_{n+1}\right)=0_{K}$. Now, we have

$$
\begin{aligned}
d\left(x_{n+p}, x_{n}\right) & \leq_{\oplus} \quad \psi^{n}\left(d\left(x_{1}, x_{0}\right)\right) \oplus \psi^{n+1}\left(d\left(x_{1}, x_{0}\right)\right) \oplus \cdots \oplus \psi^{n+p-1}\left(d\left(x_{1}, x_{0}\right)\right) \\
\leq_{\oplus} & \psi^{n}\left(d\left(x_{1}, x_{0}\right)\right) \oplus \psi^{n}\left(d\left(x_{1}, x_{0}\right)\right) \oplus \cdots \oplus \psi^{n+p-2}\left(d\left(x_{1}, x_{0}\right)\right) \\
& \cdot \\
& \cdot \\
& . \\
& \\
& \\
& =\psi^{n}\left(d\left(x_{1}, x_{0}\right)\right) \oplus \psi^{n}\left(d\left(x_{1}, x_{0}\right)\right) \oplus \cdots \oplus \psi^{n}\left(d\left(x_{1}, x_{0}\right)\right) \\
&
\end{aligned}
$$


Since $\psi^{n}(a)$ is convergent to $0_{K}$ for each $a \in K^{+},\left\{x_{n}\right\}$ is a Cauchy sequence in the metric space $X$, we have $\lim _{n, m \rightarrow \infty} d\left(x_{m}, x_{n}\right)=0_{K}$, Since $X$ is complete, then $\left\{x_{n}\right\}$ is convergent to $x$. Therefore,

$$
d(x, x)=\lim _{n \rightarrow \infty} d\left(x_{n}, x\right)=\lim _{n, m \rightarrow \infty} d\left(x_{m}, x_{n}\right)=0_{K} .
$$

Now, we claim that $T x=x$. We show that $d(x, T x)=0_{K}$. Assume this is not true. From we obtain

$$
\begin{aligned}
d(x, T x) & \leq_{\oplus} d\left(x, T x_{n}\right) \oplus d\left(T x_{n}, T x\right) \\
& \leq_{\oplus} d\left(x, T x_{n}\right) \oplus \psi\left(\max \left\{d\left(x, x_{n}\right), d(x, T x), d\left(x_{n}, x_{n+1}\right)\right\}\right) .
\end{aligned}
$$

using the continuity of and letting $n \rightarrow \infty$, we have

$$
d(x, T x) \leq_{\oplus} \psi(d(x, T x))<_{\oplus} d(x, T x) .
$$

which is a contradiction. Thus $d(x, T x)=0$ and so $x=T x$. Now let $z$ is another fixed point of $T$, that is $x \neq z$, then from (3.1) we have

$$
\begin{aligned}
d(x, z)=d(T x, T x)) & \leq_{\oplus} \psi(\max \{d(x, z), d(x, T x), d(z, T z)\}) \\
& =\psi(\max \{d(x, z), d(x, x), d(z, z)\}) \\
& =\psi(d(x, z)) \\
& <_{\oplus} d(x, z) .
\end{aligned}
$$

which is a contradiction. Thus $x=z$.

Definition 3.2. Suppose that $(X, K, d)$ be a partial idempotent-valued metric space. We call a mapping $T: X \rightarrow X$ is a idempotent-valued contractive mapping on $X$, if there exists an $a \in \mathbb{R}$ with $a \in[0,1)$ such that

$$
d(T x, T y) \leq_{\oplus} a d(x, y), \text { for } x, y \in X .
$$

Example 3.3. Let $X=(-\infty, 0], K=\mathbb{R}$ with the operations $a \oplus_{\min } b:=\min \{a, b\}$. Define the metric $d: X \times X \rightarrow K^{+}$by $d(a, b):=a \oplus b$. The map $T: X \rightarrow X$ defined by $T x=\frac{1}{2} x$, for $x \in X$ is a idempotent-valued contractive mapping on $X$, i.e we have

$$
d(T x, T y)=\min \left\{\frac{1}{2} x, \frac{1}{2} y\right\} \leq_{\oplus} \frac{1}{2} \min \{x, y\}=\frac{1}{2} d(x, y) .
$$

Theorem 3.4. Let $(X, K, d)$ be a complete partial idempotent -valued metric spaces and let $K$ be a normal space. If $T$ be a contractive mapping on $X$. Then $T$ has at least one fixed point

Proof. It is a Consequence of Theorem 3.1. In fact, if we take $\psi(k)=a k$ where $a \in[0,1)$, $k \in K^{+}$, we have Theorem 3.4. 
Example 3.5. Let $K=\mathbb{R}^{2}$ with partial order which is defined in Example $2.8, X=\mathbb{R}^{2^{+}}$ with the operations

$$
(a, b) \oplus(c, d):=\max \{(a, b),(c, d)\} .
$$

And the metric $d: X \times X \rightarrow K$ defined by

$$
d(x, y)=x \oplus y, \text { for } x, y \in X
$$

Now define the mapping $T: X \rightarrow X$ by

$$
T\left(\left(x_{1}, x_{2}\right)\right)=\left(\frac{1}{2} x_{1}, \frac{1}{5} x_{2}\right) .
$$

It is clear that

$$
d(T(x), T(y)) \leq_{\oplus} \frac{1}{2} d(x, y) .
$$

By above theorem $T$ has a unique fixed point in $X$.

In the following theorem, we give fixed point theorem on partial ordered spaces. The contractive condition (3.1) does not have to be satisfied for $x, y \in X$, but we add a condition on $X$.

Theorem 3.6. Let $(X, \leq)$ be complete partial idempotent-valued metric space and $T: X \rightarrow$ $X$ be a continuous, nondecreasing mapping such that

$$
d(T x, T y) \leq_{\oplus} \psi(\max \{d(x, T x), d(x, y), d(y, T y)\}) .
$$

for all $x, y \in X$ with $y \leq x$, where $\psi: K^{+} \rightarrow K^{+}$is a continuous, nondecreasing function such that $\lim _{n \rightarrow \infty} \psi^{n}(a)=0_{K}$ and $\psi(a)<_{\oplus}$ a for $a \in K^{+}$. If there exists an $x_{0} \in X$ with $x_{0} \leq T x_{0}$, then there exists $x \in X$ such that $x=T x$.

Proof. Let $x_{n}=T x_{n-1}$ for $n=1,2, \cdots$ If $x_{n_{0}}=x_{n_{0}-1}$ for some $n_{0} \in N$, then it is clear that $x_{n_{0}}$ is a fixed point of $T$. Thus, assume $x_{n_{0}} \neq x_{n_{0}-1}$ for all $n$. Notice that since $x_{0} \leq T x_{0}$ and $T$ is nondecreasing, we have

$$
x_{0}<x_{1}<x_{2}<\cdots<x_{n} \cdots
$$

Now, since $x_{n-1}<x_{n}$, we can use the inequality (3.2) for these points, similar to proof Theorem 3.1 we can show that $x_{n}$ is a Cauchy sequence in the metric space $X$, we have $\lim d\left(x_{m}, x_{n}\right)=0_{K}$, Since $X$ is complete, then $x_{n}$ is convergent to $x$. Therefore,

$$
d(x, x)=\lim _{n \rightarrow \infty} d\left(x_{n}, x\right)=\lim _{n, m \rightarrow \infty} d\left(x_{m}, x_{n}\right)=0_{K} .
$$


Now, we claim that $T x=x$. Suppose $d(x, T x)>_{\oplus} 0_{K}$. Since $T$ is continuous, therefore $d(T x, T x)=\lim _{n \rightarrow \infty} d\left(T x_{n}, T x\right)$. Now from (3.2) we have

$$
d(T x, T x) \leq_{\oplus} \psi(\max \{d(x, T x), d(x, x)\})=\psi(d(x, T x)) .
$$

As

$$
d(x, T x) \leq_{\oplus} d\left(x, x_{n+1}\right) \oplus d\left(x_{n+1}, T x\right),
$$

and letting $n \rightarrow \infty$, we have

$$
\begin{aligned}
d(x, T x) & \leq_{\oplus} \lim _{n \rightarrow \infty} d\left(x, x_{n+1}\right) \oplus \lim _{n \rightarrow \infty} d\left(x_{n+1}, T x\right) \\
& =d(T x, T x) \\
& \leq_{\oplus} \psi(d(x, T x) \\
& <_{\oplus} d(x, T x) .
\end{aligned}
$$

which is a contradiction since $d(x, T x) \geq_{\oplus} 0_{K}$. Thus $d(x, T x)=0_{K}$, therefore $x=T x$.

Example 3.7. Let $X=\mathbb{R}^{+}, K=\mathbb{R}$ and $d(x, y)=x \oplus y=\max \{x, y\}$. We can define a partial order on $X$ as follows:

$$
x \preceq y \Longleftrightarrow y=x \text { or }\{x, y \in[0,1] \text { with } x \leq y\} .
$$

Let

$$
T(x)=\left\{\begin{aligned}
\frac{x^{2}}{1+x} & x \in[0,1] \\
2 x & \text { o.w }
\end{aligned}\right.
$$

and $\psi: \mathbb{R}^{+} \longrightarrow \mathbb{R}^{+}, \psi(t)=\frac{t}{1+t}$. Therefore, $\psi$ is continuous and nondecreasing. Also, $T$ is nondecreasing with respect to $\preceq$ and for $y \prec x$, we have

$$
\begin{aligned}
d(T x, T y) & =\max \left\{\frac{x^{2}}{1+x}, \frac{y^{2}}{1+y}\right\} \\
& =\frac{x^{2}}{1+x} \\
\leq_{\oplus} & \psi(\max \{d(x, T x), d(x, y), d(y, T y)\}) .
\end{aligned}
$$

It is clear that the conditions of Theorem 3.6 is satisfied and for $x_{0}=0$, we have $x_{0} \leq T x_{0}$. Therefore by Theorem 3.6, $T$ has a fixed point in $X$.

This example shows that we can not use of Theorem 2.1 in [3] because $\sum \psi^{n}(t)$ is not convergent.

For each $x \in X$ and $0_{K}<r$, let $B(x, r)=\left\{y \in X: d(x, y)<_{\oplus} r\right\}$ and $B[x, r]=\{y \in X:$ $\left.d(x, y) \leq_{\oplus} r\right\}$. As in the real case, the equality $B[x, r]=\overline{B(x, r)}$ does not necessarily hold in general case. In the next lemma we show this equality in idempotent -valued metric spaces. 
Lemma 3.8. Let $(X, K, d)$ be a partial idempotent-valued metric space and let $K$ be a normal space. Then for $x \in X$ and $0_{K}<r, B[x, r]=\overline{B(x, r)}$

Proof. For each $y \in B[x, r]$, if $d(x, y)<_{\oplus} r$ then $y \in B(x, r) \subseteq \overline{B(x, r)}$. If $d(x, y)=r$, then let $\varepsilon_{n}=\frac{r}{n}$ and $z_{n} \in X$ such that $d\left(z_{n}, y\right)<\varepsilon_{n}$. Since

$$
d\left(x, z_{n}\right)<_{\oplus} \frac{r}{n} \oplus r \leq_{\oplus} r \oplus r=r,
$$

then $z_{n} \in B(x, r)$. Since $d(y, y) \leq d\left(y, z_{n}\right)=\frac{r}{n} \rightarrow 0_{K}$ therefore $\lim _{n \rightarrow \infty} z_{n}=y$. This implies $y \in \overline{B(x, r)}$.

Corollary 3.9. Let $(X, K, d)$ be a complete partial idempotent-valued metric space and let $K$ be a normal space and

$$
B\left(y_{0}, r\right):=\left\{x \in X: d\left(x, y_{0}\right)<_{\oplus} r\right\},
$$

where $r \in K$ and $r>_{\oplus} 0_{K}$. Suppose $T: B\left(y_{0}, r\right) \rightarrow X$ be a idempotent-valued contractive mapping, such that

$$
d\left(T y_{0}, y_{0}\right)<_{\oplus} b r .
$$

where $b \in[0,1)$. Then $T$ has a fixed point.

Proof. We show that $T\left(\overline{B\left(y_{0}, r\right)}\right) \subseteq \overline{B\left(y_{0}, r\right)}$. Let $x \in \overline{B\left(y_{0}, r\right)}$. Then $d\left(x, y_{0}\right) \leq_{\oplus} r$ and

$$
d\left(T x, y_{0}\right) \leq_{\oplus} d\left(T x, T y_{0}\right) \oplus d\left(T y_{0}, y_{0}\right) \leq_{\oplus} a d\left(x, y_{0}\right) \oplus b r .
$$

we obtain

$$
d\left(T x, y_{0}\right) \leq_{\oplus} a r \oplus b r \leq_{\oplus} r \oplus r=r,
$$

which shows that $T x \in \overline{B\left(y_{0}, r\right)}$. We can apply Theorem 3.1 to get the conclusion.

\section{Best Proximity Point Results}

In the light of extending the technique in the previous section to obtain best proximity point results in normal partial idempotent-valued space, we recall some notions and notations. Whole this section, $K$ is supposed that is a normal space.

Definition 4.1. Let $A$ and $B$ be non-empty subsets of a partial idempotent-valued metric space $X$, we denote the distance between $A$ and $B$ by

$$
\operatorname{dist}(A, B):=\inf \{d(a, b): a \in A, b \in B\} .
$$

Definition 4.2. Let $A$ and $B$ be non-empty subsets of a partial idempotent-valued metric space $X$. A map $T: A \cup B \rightarrow A \cup B$ is a cyclic contraction map if it satisfies: 
(1) $T(A) \subseteq B$ and $T(B) \subseteq A$.

(2) For some $a \in\left(\frac{1}{2}, 1\right)$ we have

$$
d(T x, T y) \leq_{\oplus} a \max \{d(x, T x), d(x, y), d(y, T y)\} \oplus(1-a) \operatorname{dist}(A, B),
$$

for all $x \in A, y \in B$.

Definition 4.3. A point $x \in A \cup B$ is called a best proximity point if $d(x, T x)=\operatorname{dist}(A, B)$.

Lemma 4.4. Let $A$ and $B$ be non-empty subsets of a normal complete partial idempotentvalued metric space $X$. Suppose $T: A \cup B \rightarrow A \cup B$ is a cyclic contraction map. Then starting with any $x_{0} \in A \cup B$, we have $d\left(x_{n}, T x_{n}\right) \rightarrow \operatorname{dist}(A, B)$ where $x_{n+1}=T x_{n}$.

Proof. We have

$$
\begin{aligned}
d\left(x_{n}, x_{n+1}\right) & \leq_{\oplus} a \max \left\{d\left(x_{n-1}, T x_{n-1}\right), d\left(x_{n-1}, x_{n}\right), d\left(x_{n}, T x_{n}\right\} \oplus(1-a) \operatorname{dist}(A, B)\right. \\
& =a \max \left\{d\left(x_{n-1}, x_{n}\right), d\left(x_{n}, x_{n+1}\right)\right\} \oplus(1-a) \operatorname{dist}(A, B)
\end{aligned}
$$

Now, if

$$
\max \left\{d\left(x_{n-1}, x_{n}\right), d\left(x_{n}, x_{n+1}\right)\right\}=d\left(x_{n}, x_{n+1}\right) .
$$

Then

$$
\begin{aligned}
d\left(x_{n}, x_{n+1}\right) & \leq \quad a d\left(x_{n}, x_{n+1}\right) \oplus(1-a) \operatorname{dist}(A, B) \\
& =a d\left(x_{n}, x_{n+1}\right) \\
& <d\left(x_{n}, x_{n+1}\right) .
\end{aligned}
$$

which is a contradiction. Thus

$$
\max \left\{d\left(x_{n-1}, x_{n}\right), d\left(x_{n}, x_{n+1}\right)\right\}=d\left(x_{n}, x_{n-1}\right),
$$

for all $n$. Therefore we have

$$
\begin{aligned}
d\left(x_{n}, x_{n+1}\right) & \leq_{\oplus} a d\left(x_{n-1}, x_{n}\right) \oplus(1-a) \operatorname{dist}(A, B) \\
& \leq_{\oplus} a\left(a d\left(x_{n-2}, x_{n-1}\right) \oplus(1-a) \operatorname{dist}(A, B)\right) \oplus a \operatorname{dist}(A, B) \\
& \leq_{\oplus} a\left(a d\left(x_{n-2}, x_{n-1}\right) \oplus a \operatorname{dist}(A, B)\right) \oplus a \operatorname{dist}(A, B) \\
& \leq_{\oplus} a\left(a d\left(x_{n-2}, x_{n-1}\right) \oplus a d\left(x_{n-2}, x_{n-1}\right)\right) \oplus a \operatorname{dist}(A, B) \\
& \leq_{\oplus} a^{2} d\left(x_{n-2}, x_{n-1}\right) \oplus \operatorname{dist}(A, B) .
\end{aligned}
$$

Inductively, we have

$$
d\left(x_{n}, x_{n+1}\right) \leq_{\oplus} a^{n} d\left(x_{0}, x_{1}\right) \oplus \operatorname{dist}(A, B) .
$$

Therefore $d\left(x_{n}, x_{n+1}\right) \rightarrow \operatorname{dist}(A, B)$. 
Proposition 4.5. Let $A$ and $B$ be non-empty closed subsets of a normal complete partial idempotent-valued metric space $X$. Let $T: A \cup B \rightarrow A \cup B$ be a cyclic contraction map, let $x_{0} \in A$ and define $x_{n+1}=T x_{n}$. Suppose $\left\{x_{2 n}\right\}$ has a convergent subsequence in $A$. Then there exists $x$ in $A$ such that $d(x, T x)=\operatorname{dist}(A, B)$.

Proof. Let $\left\{x_{2 n_{k}}\right\}$ be a subsequence of $\left\{x_{2 n}\right\}$ converging to some $x \in A$. Now

$$
\operatorname{dist}(A, B) \leq_{\oplus} d\left(x, x_{2 n_{k}-1}\right) \leq_{\oplus} d\left(x, x_{2 n_{k}}\right) \oplus d\left(x_{2 n_{k}}, x_{2 n_{k}-1}\right) \rightarrow \operatorname{dist}(A, B) .
$$

Since

$$
\operatorname{dist}(A, B) \leq_{\oplus} d\left(x_{2 n_{k}}, T x\right) \leq_{\oplus} d\left(x_{2 n_{k}-1}, x\right) \rightarrow \operatorname{dist}(A, B) .
$$

Thus $d(x, T x)=\operatorname{dist}(A, B)$.

Corollary 4.6. Let $A$ and B be non-empty closed subsets of a normal complete partial idempotent-valued metric space $(X, d)$ and let $T: A \cup B \rightarrow A \cup B$ be a cyclic contraction. If either $A$ or $B$ is sequentially compact then there exists $x \in A \cup B$ with $d(x, T x)=\operatorname{dist}(A, B)$.

Proof. It is a consequence of Proposition 4.5.

Definition 4.7. Let $A$ and $B$ be non-empty subsets of a partial idempotent-valued metric space $(X, d)$. The ordered pair $(A, B)$ is said to satisfy the property $U C$ if the following holds:

If $\left\{x_{n}\right\}$ and $\left\{z_{n}\right\}$ are sequences in $A$ and $\left\{y_{n}\right\}$ be a sequence in $B$ such that $d\left(x_{n}, y_{n}\right) \rightarrow$ $\operatorname{dist}(A, B)$ and $d\left(z_{n}, y_{n}\right) \rightarrow \operatorname{dist}(A, B)$ then $d\left(x_{n}, z_{n}\right) \rightarrow 0_{K}$.

Definition 4.8. Let $A$ and $B$ be non-empty subsets of a partial idempotent-valued metric space $(X, d)$. The ordered pair $(A, B)$ is said to satisfy the property $U C^{*}$ if $(A, B)$ has property $U C$ and the following condition holds: If $\left\{x_{n}\right\}$ and $\left\{z_{n}\right\}$ are sequences in $A$ and $\left\{y_{n}\right\}$ is a sequence in $B$ satisfying:

(i) $d\left(z_{n}, y_{n}\right) \rightarrow \operatorname{dist}(A, B)$ as $n \rightarrow \infty$.

(ii) For each $0_{K}<_{\oplus} \varepsilon$ there exists $n_{0} \in \mathbb{N}$ such that $d\left(z_{n}, y_{n}\right) \rightarrow \operatorname{dist}(A, B) \oplus \varepsilon$ for all $n>n_{0}$,

Then $d\left(x_{n}, z_{n}\right) \rightarrow 0_{K}$.

Example 4.9. Every pair of non-empty subsets $A, B$ of a idempotent-valued metric space $(X, d)$ such that $d(A, B)=0$ has the property $U C^{*}$.

Theorem 4.10. Let $A$ and $B$ be non-empty subsets of a normal complete partial idempotentvalued metric space $(X, d)$. The ordered pair $(A, B)$ has the property $U C^{*}$. Suppose $T$ : $A \cup B \rightarrow A \cup B$ is a cyclic contraction map, then there exists a best proximity point $x$ in $A$ (that is with $d(x, T x)=\operatorname{dist}(A, B)$. Further, if $x_{0} \in A$ and $x_{n+1}=T x_{n}$ then $\left\{x_{2 n}\right\}$ converges to the best proximity point. 
Proof. Since the pair $(A, B)$ has the property $U C^{*}$ and $d\left(x_{2 n}, T x_{2 n}\right) \rightarrow \operatorname{dist}(A, B), d\left(T^{2} x_{2 n}, T x_{2 n}\right) \rightarrow$ $\operatorname{dist}(A, B)$ we have

$$
d\left(x_{2 n}, x_{2 n+1}\right) \rightarrow 0_{K}
$$

Also

$$
d\left(T x_{2 n}, T x_{2 n+1}\right) \rightarrow 0_{K}
$$

We now show that for every $0_{K}<_{\oplus} \varepsilon$ there exists $n_{0}$ such that for all $m>n>n_{0}$,

$$
d\left(x_{2 m}, T x_{2 n}\right) \leq_{\oplus} \operatorname{dist}(A, B) \oplus \varepsilon
$$

Suppose not, then there exists $0_{K}<_{\oplus} \varepsilon$ such that for all $k \in \mathbb{N}$ there exists $m_{k}>n_{k} \geq k$ for which

$$
\operatorname{dist}(A, B) \oplus \varepsilon \leq_{\oplus} d\left(x_{2 m}, T x_{2 n}\right),
$$

this $m_{k}$ can be chosen such that it is the least integer greater than $n_{k}$ to satisfy the above inequality. Now

$$
\begin{aligned}
\operatorname{dist}(A, B) \oplus \varepsilon & \leq_{\oplus} d\left(x_{2 m_{k}}, T x_{2 n_{k}}\right) \\
& \leq_{\oplus} d\left(x_{2 m_{k}}, x_{2 m_{k-1}}\right) \oplus d\left(x_{2 m_{k-1}}, T x_{2 n_{k}}\right) \\
& \leq_{\oplus} \quad \operatorname{dist}(A, B) \oplus \varepsilon
\end{aligned}
$$

Hence $\lim _{m \rightarrow \infty} d\left(x_{2 m}, T x_{2 n}\right) \rightarrow \operatorname{dist}(A, B) \oplus \varepsilon$. Consequently,

$$
\begin{aligned}
d\left(x_{2 m_{k}}, T x_{2 n_{k}}\right) & \leq_{\oplus} d\left(x_{2 m_{k}}, x_{2 m_{k+1}}\right) \oplus d\left(x_{2 m_{k+1}}, T x_{2 n_{k+1}}\right) \\
& \oplus d\left(T x_{2 n_{k+1}}, T x_{2 n_{k}}\right) \\
& \leq_{\oplus} d\left(x_{2 m_{k}}, x_{2 m_{k+1}}\right) \oplus a^{2} d\left(x_{2 m_{k}}, x_{2 n_{k}}\right) \oplus \operatorname{dist}(A, B) \\
& \oplus d\left(T x_{2 n_{k+1}}, T x_{2 n_{k}}\right) .
\end{aligned}
$$

Hence

$$
\begin{aligned}
\operatorname{dist}(A, B) \oplus \varepsilon & \leq_{\oplus} \quad a^{2}(\operatorname{dist}(A, B) \oplus \varepsilon) \oplus \operatorname{dist}(A, B) \\
& <\oplus \quad(\operatorname{dist}(A, B) \oplus \varepsilon) \oplus \operatorname{dist}(A, B) \\
& =\quad \operatorname{dist}(A, B) \oplus \varepsilon .
\end{aligned}
$$

which is a contradiction. Since the ordered pair $(A, B)$ has the property $U C^{*}$ then $\left\{x_{2 n}\right\}$ is a Cauchy sequence and hence converges to some $x \in A$. From Proposition 4.5 it follows that $d(x, T x)=\operatorname{dist}(A, B)$. 


\section{References}

[1] M. ABBAS, G. JUNGCK, Common fixed points results for noncommuting mapping without continuity in cone metric space, J. Math. Anal. Appl. 341, (2008) 416-420.

[2] I. Altun, F. Sola, H. Simsek,Generalized contractions on partial metric spaces, Topol. Appl. 157 (18) (2010) 2778-2785.

[3] I. Altun, A. ERduran, Fixed point theorems for monotone mappings on partial metric spaces, Fixed Point Theory Appl. 2011, Article ID 508730 (2011).

[4] J. Caballero, J. Harjani, K. Sadarangani, Contractive-like mapping principles in ordered metric spaces and applications to ordinary differential equations, Fixed Point Theory Appl. 2010, Article ID 916064 (2010).

[5] A. A. Eldred, P. Veeramani, Existence and convergence of best proximity points, J. Math. Anal. Appl. 323, (2006) 1001-1006.

[6] LG. HuAng, X. ZHAng, Cone metric spaces and fixed point theorems of contractive mappings, J. Math. Anal. Appl. 332(2), (2007) 1468-1476.

[7] C. Mongkolkeha, P. Kumam, Best proximity point theorems for generalized cyclic contractions in ordered metric spaces, J. Opt. Theory Appl. 155 (1) (2012), 215-226,

[8] S.G. Matthews, Partial metric topology, Research Report 212, Department of Computer Science, University of Warwick, 1992.

[9] Z. Mustafa, H. Huang, S. Radenović, Some fixed point generalizations are not real generalizations, J. Adv. Math. Stud. 9, (2016), 110-116.

[10] S. Oltra, O. VALERo, Banach fixed point theorem for partial metric spaces, Rendiconti dell Istituto di Matematica dell-Universit di Trieste, 36 (12), (2004) 17-26.

[11] D. O'Regan, A. Petrusel, Fixed point theorems for generalized contractions in ordered metric spaces, J. Math. Anal. Appl. 341 (2), (2008) 1241-1252.

[12] JB. PROLLA, Fixed point theorems for set valued mappings and existence of best approximations, Numer Funct Anal Optim,5, (1982-1983) 449-455.

[13] S. RAdENOVIĆ, COMmON FIXEd POINTS UNDER CONTRACTIVE CONDITIONS IN CONE METRIC SPACES, Comput. Math. Appl. 58 (2009) 123-1278.

[14] S. Radenović, V. Rakoćević, SH. Rezapour, Common fixed points for $(g, f)$ type maps in cone metric spaces, Appl. Math.Comput. 218, (2011) 480-491.

[15] H. Rahimi, B. E. Rhoades, S. Radenović, G. Soleimani Rad, Fixed and periodic point theorems for T-contractions on cone metric spaces, Filomat, 27 (5) (2013), 881-888.

[16] S. REICH, Approximate selections, best approximations, fixed points and invariant sets, J. Math. Anal App, 62, (1978)104-113.

[17] VM. Sehgal, SP. Singh, A generalization to multifunctions of Fans best approximation theorem, Proc Am Math Soc, 102, (1988) 534-537.

[18] S. SADIQ BASHA, Best proximity point theorems generalizing the contraction principle, Nonlinear Anal. 74, (2011) 5844-5850. 
[19] V. Vetrivel, P. Veeramani, P. Bhattacharyya, Some extensions of Fans best approxomation theorem, Numer Funct Anal Optim. 13, (1992) 397-402.

[20] QL. XIN, LN. JIANG, Fixed point theorems for mappings satisfying the ordered contractive condition on noncommutative spaces, Fixed Point Theory Appl. 2014, Article ID 30 (2014).

[21] S. Xu, Ć. DolićAnin, S. RAdenović, Some rematks on results of Perov type, J. Adv. Math. Stud. 13 (3), (2016), 361-369

[22] JC.Yeol, S. ReZA, SH. WANG, Common fixed point theorems on generalized distance in ordered cone metric spaces, Comput. Math. Appl. 61,(2011) 1254-1260. 